\title{
Anisotropic interaction of two-level systems with acoustic waves in disordered crystals
}

\author{
Dragoş-Victor Anghel ${ }^{1}$ and Dmitry Churochkin ${ }^{2}$ \\ ${ }^{1}$ Department of Theoretical Physics, National Institute \\ for Physics and Nuclear Engineering-"Horia Hulubei", \\ Str. Atomistilor no.407, P.O.BOX MG-6, Bucharest-Magurele, Romania \\ ${ }^{2}$ Saratov State University, 410012, Astrakhanskaya St. 83, Saratov, Russia
}

(Dated: October 26, 2018)

\begin{abstract}
We apply the model introduced in Phys. Rev. B 75, 064202 (2007), cond-mat/0610469, to calculate the anisotropy effect in the interaction of two level systems with phonons in disordered crystals. We particularize our calculations to cubic crystals and compare them with the available experimental data to extract the parameters of the model. With these parameters we calculate the interaction of the dynamical defects in the disordered crystal with phonons (or sound waves) propagating along other crystalographic directions, providing in this way a method to investigate if the anisotropy comes from the two-level systems being preferably oriented in a certain direction or solely from the lattice anisotropy with the two-level systems being isotropically oriented.

PACS numbers: 61.43.-j,62.40.+i,63.20.kp
\end{abstract}

\section{INTRODUCTION}

The low temperature acoustic and thermal properties of amorphous, glassy materials are remarkably similar and they can be explained to a large extent by assuming that the material contains a large number of dynamic defects. These dynamic defects are tunneling systems (TS) and are modeled by an ensemble of two-level systems (TLS) [1, 2]. Crystals with defects-with a large enough amount of disorder-exhibit also glass-like properties, but these properties are not so universal and, even more, they are not isotropic-like it is, for example, the sound absorbtion and velocity change, which depends on the crystalographic direction in which the sound propagates [3]. 
Since a detailed microscopic model of tunneling systems in glassy materials is still not available, the study of disordered crystals is especially interesting because it offers an additional opportunity for their clarification: in some materials we know quite well which are the entities that tunnel between different equilibrium positions. Beside this, the anisotropy of the TLS-sound wave interaction in crystals represents another challange to the interaction models of TLSs which requires clarification.

In this paper we give an explanation for the anisotropy observed in the glass-like properties of general, disordered crystals, by employing a model recently published [4]. In this model we assume that each TLS is characterized by a direction in space, call it $\hat{\mathbf{t}}$-this might be the direction defined by the two potential wells of the tunneling system, or the axis of rotation of the tunneling entity-and we introduce a coupling between the TLS and a strain field $[S]$, which is dependent on the amplitude of $[S]$ at the position of the TLS and on the orientation of $\hat{\mathbf{t}}$ with respect to $[S]$. In [4] the model was applied to an amorphous solid, assuming that the directions $\hat{\mathbf{t}}$ are isotropically distributed, and the effective coupling of an elastic wave with a TLS was calculated as the average over the directions of the TLS. In this way it was proven on very general grounds that, on average, the longitudinal waves couple with the TLSs stronger than the transversal waves-in standard notations, $\gamma_{l}>\gamma_{t}[4]$.

In a disordered crystal with TLSs, there could be at least two sources of anisotropy. The first one is that the TLSs might not be anymore isotropically oriented so the effective coupling of elastic waves with them depends, through $\hat{\mathbf{t}}$, on the waves direction of propagation and on their polarization. The second source of anisotropy is that besides the relative orientation of $\hat{\mathbf{t}}$ and $[S]$, the symmetry of the crystal is manifested also in the interaction of elastic waves with TLSs [4, 5]. This leads to anisotropy effects in the interaction of elastic waves with TLS even if the TLS distribution is isotropic.

In this paper we shall analyse mainly the second type of anisotropy. We shall assume that the TLS orientations are isotropically distributed and we shall calculate the anisotropy effects imposed only by the lattice symmetries onto the interaction hamiltonian. Existing experimental data (see Ref. [6] and references therein) will enable us to obtain relations between the parameters of the model for a cubic lattice. Using these parameters we can make predictions about the absorbtion of elastic waves propagating along other crystalographic directions or having different polarizations. These predictions could constitute a first test for the isotropy of the TLS orientations in a specific crystal. 


\section{ANISOTROPIC INTERACTION OF TWO-LEVEL SYSTEMS WITH SOUND WAVES}

In the standard tunneling model (STM), the Hamiltonian of an isolated TLS is written in a two-dimensional basis as [1, 2]

$$
H_{\mathrm{TLS}}=\frac{\Delta}{2} \sigma_{z}-\frac{\Lambda}{2} \sigma_{x} \equiv \frac{1}{2}\left(\begin{array}{cc}
\Delta & -\Lambda \\
-\Lambda & -\Delta
\end{array}\right)
$$

where $\Delta$ is called the asymmetry of the potential and $\Lambda$ the tunnel splitting. The basis in which is written the Hamiltonian of Eq. (11) is chosen in such a way that a perturbation to the TLS, caused by a strain field, say $[S]$, is described by a diagonal Hamiltonian,

$$
H_{1}=\frac{1}{2}\left(\begin{array}{cc}
\delta & 0 \\
0 & -\delta
\end{array}\right) .
$$

with $\delta \equiv 2[\gamma]:[S]$ and $[\gamma]$ a second rank tensor of coupling constants; by "." we denote the dyadic product. Typically, in the STM one considers the coupling of TLSs with transversally or longitudinally polarized sound waves, so not too much attention has been given to the $[\gamma]$ tensor and in general $\delta$ is written simply as $\delta=2 \gamma_{l, t} S_{l, t}$, with $\gamma$ and $S$ being scalars ( $S$ is the amplitude of the strain field) and $l$ and $t$ denoting the longitudinal $(l)$ or the transversal $(t)$ polarization of the sound wave, respectively. Such a simple description of the TLS-strain field interaction has several shortcomings-e.g. $\delta$ is not invariant and even leads to physical ambiguities at the rotation of the coordinates axes-and cannot account for the different coupling of the TLS with sound waves propagating in different directions. In consequence, in Ref. [4] it is proposed a model which eliminates the shortcomings and takes into account the symmetries of the material in which the TLSs are embeded and the orientation of the TLS with respect to the strain field. Let us describe briefly how this is done.

We construct form the components of $\hat{\mathbf{t}}$ the simple $3 \times 3$ symmetric tensor, [T], of components $T_{i j}=t_{i} t_{j}$ and we introduce the forth rank tensor of TLS-strain field coupling constants, $[[R]]$. With these two objects, we build the general tensor $[\gamma]$, as $\gamma_{i j}=T_{k l} R_{k l i j}$-throughout this paper we assume summation over the repeated indices. The forth rank tensor $[[R]]$ has a similar structure as the forth rank tensor $[[c]]$ of stifness constants and reflects the symmetries of the crystal that contains the TLS [4, 7].

For the convenience of the calculations we work here, like in Refs. [4, 5, 7], in abbreviated subscript notations and write $[T]$ and $[S]$ as the six elements vectors, $\mathbf{T} \equiv$ 
$\left(T_{11}, T_{22}, T_{33}, 2 T_{23}, 2 T_{13}, 2 T_{12}\right)^{t}$ and $\mathbf{S} \equiv\left(S_{11}, S_{22}, S_{33}, 2 S_{23}, 2 S_{13}, 2 S_{12}\right)^{t}$, where by ".t" we denote the transpose. Following the notations of Auld [8], the components of the symmetric tensors will be denoted in abbreviated subscript notations by a single, upper case subscripte.g. $T_{I}, S_{I}$, and $T_{3} \equiv T_{33}=t_{3}^{2}$; also in abbreviated subscript notations, the tensors $[[R]]$ and $[[c]]$ will be written as $6 \times 6$ matrices, $[R]$ and $[c]$, of components $R_{I J}$ and $c_{I J}$, respectively. Putting all these together we get the expression $\delta=2 \mathbf{T}^{t} \cdot[R] \cdot \mathbf{S}[4,5$, 7].

Having now the full expression for the interaction Hamiltonian, $H_{1}$, we can calculate the amplitude of excitation of a TLS, of parameters $\Delta$ and $\Lambda$, by a phonon of wave-vector $\mathbf{k}$ and polarization $\sigma$; we denote by $n_{\mathbf{k} \sigma}$ the number of phonons on the mode $(\mathbf{k}, \sigma)$ after the TLS excitation process. The displacement field of the phonon, $\mathbf{u}_{\mathbf{k} \sigma}$, is normalized to $N_{\mathbf{k} \sigma} \equiv \sqrt{\hbar /\left(2 V \rho \omega_{\mathbf{k} \sigma}\right)}$, and has the strain field $\mathbf{S}_{\mathbf{k} \sigma}=\nabla_{S} \mathbf{u}_{\mathbf{k} \sigma}$ (where by $\nabla_{S}$ we denote the symmetric gradient). This way we get

$$
\left\langle n_{\mathbf{k} \sigma}, \uparrow\left|\tilde{H}_{1}\right| n_{\mathbf{k} \sigma}+1, \downarrow\right\rangle_{\mathbf{u}_{\mathbf{k} \sigma}}=-\frac{\Lambda}{\epsilon} \sqrt{n_{\mathbf{k} \sigma}} \mathbf{T}^{t} \cdot[R] \cdot \mathbf{S}_{\mathbf{k} \sigma}
$$

where $\epsilon=\sqrt{\Delta^{2}+\Lambda^{2}}$ is the excitation energy of the TLS. Therefore the phonon scattering rate by a TLS in the ground state is

$$
\Gamma_{\mathbf{k} \sigma}(\hat{\mathbf{t}})=\frac{2 \pi}{\hbar} \frac{\Lambda^{2} n_{\mathbf{k} \sigma}}{\epsilon^{2}}\left|\mathbf{T}^{t} \cdot[R] \cdot \mathbf{S}_{\mathbf{k} \sigma}\right|^{2} \delta(\epsilon-\hbar \omega) .
$$

The main characteristic of the TLS-elastic strain interaction, is contained in the quantity $M_{\mathbf{k}, \sigma}(\hat{\mathbf{t}}) \equiv \mathbf{T}^{t} \cdot[R] \cdot \mathbf{S}_{\mathbf{k} \sigma}$, which we shall calculate next.

As mentioned above, $[R]$, like $[c]$, reflects the symmetries of the lattice. The most general type of lattice is triclinic, in which case $[R]$ is symmetric and contains 21 independent constants. Such a lattice is very complex and in general does not sustain simple transversally or longitudinally polarized elastic waves, but instead, the elastic waves propagating through the crystal will be complex superpositions of longitudinally and transversally polarized planewaves. So, to start with a simpler case and also to be able to compare our calculations with available experimental data [6], we shall focus in this paper on lattices with cubic symmetry. The tensor $[R]$ for the cubic lattice is very similar to the one for an isotropic material [4, 5, 7], but it contains 3 independent constants instead of 2 , like in the isotropic case. So we can 
preserve the notations of Refs. [4, 5, 7], and write

$$
[R]=\tilde{\gamma} \cdot\left(\begin{array}{cccccc}
1 & \zeta & \zeta & 0 & 0 & 0 \\
\zeta & 1 & \zeta & 0 & 0 & 0 \\
\zeta & \zeta & 1 & 0 & 0 & 0 \\
0 & 0 & 0 & \xi & 0 & 0 \\
0 & 0 & 0 & 0 & \xi & 0 \\
0 & 0 & 0 & 0 & 0 & \xi
\end{array}\right),
$$

without imposing the isotropy constraint, $\zeta+2 \xi=1$; similarly, the tensor of elastic stiffness constants is

$$
[c]=\left(\begin{array}{cccccc}
c_{11} & c_{12} & c_{12} & 0 & 0 & 0 \\
c_{12} & c_{11} & c_{12} & 0 & 0 & 0 \\
c_{12} & c_{12} & c_{11} & 0 & 0 & 0 \\
0 & 0 & 0 & c_{44} & 0 & 0 \\
0 & 0 & 0 & 0 & c_{44} & 0 \\
0 & 0 & 0 & 0 & 0 & c_{44}
\end{array}\right)
$$

Using $[c]$ we can write the Christoffel equation to find $\mathbf{u}$ and $\mathbf{S}$ for the elastic waves propagating in different directions and then we can calculate $M$ for any $\hat{\mathbf{t}}, \Delta$ and $\Lambda$. In the end, we average over the ensemble of TLSs, to determine the attenuation of the elastic wave or the scattering rate of the phonon. We shall apply this procedure for strain fields corresponding to elastic waves propagating along the crystalographic directions $\langle 100\rangle,\langle 110\rangle$ and $\langle 111\rangle$ of the cubic lattice. Along these directions, the cubic lattice can sustain simple, longitudinally and transversally polarized elastic waves, for any allowed values of the parameters $c_{11}, c_{12}$, and $c_{44}$.

Solving the Christoffel equations we find that the sound velocities of the longitudinal waves propagating in the $\langle 100\rangle,\langle 110\rangle$ and $\langle 111\rangle$ directions are $c_{l,\langle 100\rangle}=\sqrt{c_{11} / \rho}$, $c_{l,\langle 110\rangle}=\sqrt{\left(c_{11}+c_{12}+c_{44}\right) / \rho}$, and $c_{l,\langle 111\rangle}=\sqrt{\left(c_{11}+2 c_{12}+2 c_{44}\right) / \rho}$, respectively. Similarly, the sound velocities of the transversal waves propagating in the $\langle 100\rangle$ and $\langle 111\rangle$ directions are $c_{t,\langle 100\rangle}=\sqrt{c_{44} / \rho}$ and $c_{t,\langle 111\rangle}=\sqrt{\left(c_{11}-c_{12}-c_{44}\right) / \rho}$, respectively, whereas for the transversal waves propagating in the $\langle 110\rangle$ direction the sound velocity depends on the direction of polarization: if the wave is polarized in the $\langle 100\rangle$ direction (and perpendicular on the direction of propagation), the sound velocity is $c_{t,\langle 110\rangle}^{\langle 100\rangle}=\sqrt{c_{44} / \rho}$, and if the wave is polarized 
in the $\langle 110\rangle$ direction (and also perpendicular on the direction of propagation), the sound velocity is $c_{t,\langle 110\rangle}^{\langle 110\rangle}=\sqrt{\left(c_{11}-c_{12}-c_{44}\right) / \rho}$. Now we can calculate $M$ for these three directions of propagation.

Since the three directions, $\hat{\mathbf{x}}, \hat{\mathbf{y}}$, and $\hat{\mathbf{z}}$ are equivalent, let us take the $\langle 100\rangle$ direction as the $\hat{\mathbf{z}}$ direction. We also define $\hat{\mathbf{t}}$ by the angles $\theta$ (nutation) and $\phi$ (precession), as $\hat{\mathbf{t}} \equiv(\sin \theta \cos \phi, \sin \theta \sin \phi, \cos \theta)^{t}$. With these conventions, we get for the longitudinal wave, $\mathbf{u}_{k \hat{\mathbf{z}}, l}(\mathbf{r})=N \hat{\mathbf{z}} e^{i k \hat{\mathbf{z}} \cdot \mathbf{r}}$,

$$
M_{k \hat{\mathbf{z}}, l}=i k \tilde{\gamma} N_{k \hat{\mathbf{z}}, l}\left[\zeta+\cos ^{2} \theta(1-\xi)\right]
$$

(to simplify the expressions without reducing the clarity, we shall always drop the exponential from the expressions of $M$ and the subscripts of $N$; the implicit subscripts of $N$ are always the same as the ones of $M$ and $\mathbf{u}$ ) and for the two, reciprocally perpendicular, transversal waves, $\mathbf{u}_{k \hat{\mathbf{z}}, t, x}(\mathbf{r})=N \hat{\mathbf{x}} e^{i k \hat{\mathbf{z}} \cdot \mathbf{r}}$ and $\mathbf{u}_{k \hat{\mathbf{z}}, t, y}(\mathbf{r})=N \hat{\mathbf{y}} e^{i k \hat{\mathbf{z}} \cdot \mathbf{r}}$,

$$
\begin{aligned}
& M_{k \hat{\mathbf{z}}, t, x}=i k \tilde{\gamma} \xi N \sin (2 \theta) \cos (\phi) \\
& M_{k \hat{\mathbf{z}}, t, y}=i k \tilde{\gamma} \xi N \sin (2 \theta) \sin (\phi)
\end{aligned}
$$

For the waves propagating in the $\langle 111\rangle$ direction we get the following results. For the longitudinal wave, $\mathbf{u}_{k(\hat{\mathbf{x}}+\hat{\mathbf{y}}+\hat{\mathbf{z}}) / \sqrt{3}, l}(\mathbf{r})=N \frac{\hat{\mathbf{x}}+\hat{\mathbf{y}}+\hat{\mathbf{z}}}{\sqrt{3}} \exp \left[i k \frac{\hat{\mathbf{x}}+\hat{\mathbf{y}}+\hat{\mathbf{z}}}{\sqrt{3}} \cdot \mathbf{r}\right]$,

$$
\begin{aligned}
M_{k(\hat{\mathbf{x}}+\hat{\mathbf{y}}+\hat{\mathbf{z}}) / \sqrt{3}, l}= & N \frac{i k \tilde{\gamma}}{3}\{[2 \sin (2 \theta)(\sin \phi+\cos \phi) \\
& \left.\left.+2 \sin (2 \phi) \sin ^{2}(\theta)\right] \xi+2 \zeta+1\right\}
\end{aligned}
$$

and for the two transversal waves, $\mathbf{u}_{k(\hat{\mathbf{x}}+\hat{\mathbf{y}}+\hat{\mathbf{z}}) / \sqrt{3}, t, p_{1}}(\mathbf{r})=N \hat{p}_{1} \exp \left[i k \frac{\hat{\mathbf{x}}+\hat{\mathbf{y}}+\hat{\mathbf{z}}}{\sqrt{3}} \cdot \mathbf{r}\right]$ and $\mathbf{u}_{k(\hat{\mathbf{x}}+\hat{\mathbf{y}}+\hat{\mathbf{z}}) / \sqrt{3}, t, p_{2}}(\mathbf{r})=N \hat{p}_{2} \exp \left[i k \frac{\hat{\mathbf{x}}+\hat{\mathbf{y}}+\hat{\mathbf{z}}}{\sqrt{3}} \cdot \mathbf{r}\right]$, with polarizations $\hat{p}_{1}=\frac{-\hat{\mathbf{x}}+\hat{\mathbf{z}}}{\sqrt{2}}, \hat{p}_{2}=\frac{-\hat{\mathbf{x}}+\hat{\mathbf{y}}}{\sqrt{2}}$, we have

$$
\begin{aligned}
M_{\frac{k(\hat{\mathbf{x}}+\hat{\mathbf{y}}+\hat{\mathbf{z}})}{\sqrt{3}}, t, p_{1}}= & N \frac{i k \tilde{\gamma}}{\sqrt{6}}[2 \xi(\cos \theta-\cos \phi \sin \theta) \sin \theta \sin \phi \\
& \left.+\left(\cos ^{2} \theta-\cos ^{2} \phi \sin ^{2} \theta\right)(1-\zeta)\right]
\end{aligned}
$$

and

$$
\begin{aligned}
M_{k(\hat{\mathbf{x}}+\hat{\mathbf{y}}+\hat{\mathbf{z}}) / \sqrt{3}, t, p_{2}}= & N \frac{i k \tilde{\gamma}}{\sqrt{6}}[\sin (2 \theta)(\sin \phi-\cos \phi) \xi \\
& \left.-\sin ^{2} \theta \cos (2 \phi)(1-\zeta)\right]
\end{aligned}
$$

respectively. 
For the longitudinal wave, $\mathbf{u}_{k(\hat{\mathbf{x}}+\hat{\mathbf{y}}) / \sqrt{2}, l}(\mathbf{r})=N \frac{\hat{\mathbf{x}}+\hat{\mathbf{y}}}{\sqrt{2}} e^{i k \frac{\hat{\mathbf{x}}+\hat{\mathbf{y}}}{\sqrt{2}} \cdot \mathbf{r}}$, propagating in the $\langle 110\rangle$ direction,

$$
\begin{aligned}
M_{k(\hat{\mathbf{x}}+\hat{\mathbf{y}}) / \sqrt{2}, l}= & N \frac{i k \tilde{\gamma}}{2}\left[2 \sin (2 \phi) \sin ^{2} \theta \xi+\left(1+\cos ^{2} \theta\right) \zeta\right. \\
& \left.+\sin ^{2} \theta\right]
\end{aligned}
$$

and for the two transversal waves in the same direction, $\mathbf{u}_{k(\hat{\mathbf{x}}+\hat{\mathbf{y}}) / \sqrt{2}, t, p_{1}^{\prime}}(\mathbf{r})=$ $N \hat{p}_{1}^{\prime} \exp \left[i k \frac{\hat{\mathbf{x}}+\hat{\mathbf{y}}}{\sqrt{2}} \cdot \mathbf{r}\right]$ and $\mathbf{u}_{k(\hat{\mathbf{x}}+\hat{\mathbf{y}}) / \sqrt{2}, t, p_{2}^{\prime}}(\mathbf{r})=N \hat{p}_{2}^{\prime} \exp \left[i k \frac{\hat{\mathbf{x}}+\hat{\mathbf{y}}}{\sqrt{2}} \cdot \mathbf{r}\right]$, with polarizations $\hat{p}_{1}^{\prime}=\hat{\mathbf{z}}$, $\hat{p}_{2}^{\prime}=\frac{-\hat{\mathbf{x}}+\hat{\mathbf{y}}}{\sqrt{2}}$, we have

$$
M_{k(\hat{\mathbf{x}}+\hat{\mathbf{y}}) / \sqrt{2}, t, p_{1}^{\prime}}=N \frac{i k \tilde{\gamma} \zeta}{\sqrt{2}} \sin (2 \theta)(\sin \phi+\cos \phi)
$$

and

$$
M_{k(\hat{\mathbf{x}}+\hat{\mathbf{y}}) / \sqrt{2}, t, p_{2}^{\prime}}=N \frac{i k \tilde{\gamma}}{2} \sin ^{2} \theta \cos (2 \phi)(\zeta-1),
$$

respectively.

Now we can calculate the phonon's scattering rates, by averaging $\Gamma_{\mathbf{k} \sigma}$ of Eq. (44) over the distribution of TLS parameters, $\Delta, \Lambda, \theta$ and $\phi$ and taking into account the scattering of phonons from and into the mode $(\mathbf{k}, \sigma)$. We assume that the parameters $\Delta$ and $\Lambda$ are independent of the parameters $\theta$ and $\phi$, and their distribution is the standard $P(\Delta, \Lambda)=$ $P_{0} / \Lambda$, where $P_{0}$ is a constant [1, 2]. We change the variables $\Delta$ and $\Lambda$ into the variables $\epsilon$ and $u \equiv \Lambda / \epsilon$, with the probability distribution $P(\epsilon, u)=P_{0} /\left(u \sqrt{1-u^{2}}\right)$ and we assume that the fraction of excited TLSs, of energy $\epsilon$, is thermal and corresponds to a temperature $T: n_{\epsilon}^{(T L S)}=\left(1+e^{\epsilon / k_{\mathrm{B}} T}\right)^{-1}$. The distribution over $\theta$ and $\phi$, say $f(\theta, \phi)$, is unknown. Plugging all these quantities into the standard scattering rate calculation, we get

$$
\begin{aligned}
\tau_{\mathbf{k} \sigma}^{-1}= & \frac{P_{0} \tanh \left(\frac{\epsilon}{2 k_{\mathrm{B}} T}\right)}{2 \hbar} n_{\mathbf{k} \sigma} \int_{0}^{\pi} \sin \theta d \theta \\
& \times \int_{0}^{2 \pi} d \phi \cdot\left|M_{\mathbf{k} \sigma}[\hat{\mathbf{t}}(\theta, \phi)]\right|^{2} f(\theta, \phi) \\
\equiv & \frac{P_{0} \tanh \left(\frac{\epsilon}{2 k_{\mathrm{B}} T}\right)}{4 \pi \hbar} n_{\mathbf{k} \sigma}\left\langle\left|M_{\mathbf{k} \sigma}(\hat{\mathbf{t}})\right|^{2}\right\rangle .
\end{aligned}
$$

Now, if we would know $f(\theta, \phi)$, we could use the expressions (7), (8), and (9) for $M$, to calculate scattering rates of phonons propagating in the three different directions.

Since, as we mentioned in the Introduction, we have no microscopic model for $f(\theta, \phi)$, we shall assume that $f(\theta, \phi)$ is constant (i.e. TLSs are isotropically oriented) and comparing 
our calculations with experimental results, we shall obtain relations between the parameters $\zeta$ and $\xi$. Using this assumption and Eq. (7a), we get for the longitudinally polarized phonon propagating in the $\langle 100\rangle$ direction,

$$
\tau_{k \hat{\mathbf{z}}, l}^{-1}=\frac{3+4 \zeta+8 \zeta^{2}}{15} \cdot \frac{2 \pi P_{0} N^{2} n k^{2} \tilde{\gamma}^{2}}{\hbar} \tanh \left(\frac{\epsilon}{2 k_{\mathrm{B}} T}\right)
$$

while for the transversally polarized waves, both Eqs. (7b) and (7c) give the same result,

$$
\tau_{k \hat{\mathbf{z}}, t}^{-1}=\frac{4 \xi^{2}}{15} \cdot \frac{2 \pi P_{0} N^{2} n k^{2} \tilde{\gamma}^{2}}{\hbar} \tanh \left(\frac{\epsilon}{2 k_{\mathrm{B}} T}\right)
$$

where we dropped also the obvious subscripts of the population number, $n$.

Similarly, in the direction $\langle 111\rangle$ we get

$$
\begin{aligned}
\tau_{\frac{k(\hat{\mathbf{x}}+\hat{\mathbf{y}}+\hat{\mathbf{z}})}{\sqrt{3}}, l}^{-1}= & \frac{5+20 \zeta+20 \zeta^{2}+16 \xi^{2}}{45} \cdot \frac{2 \pi P_{0} k^{2} N^{2} n \tilde{\gamma}^{2}}{\hbar} \\
& \times \tanh \left(\frac{\epsilon}{2 k_{\mathrm{B}} T}\right),
\end{aligned}
$$

and

$$
\begin{aligned}
\tau_{\frac{k(\hat{\mathbf{x}}+\hat{\mathbf{y}}+\hat{\mathbf{z}})}{\sqrt{3}}, t}^{-1}= & \frac{2\left[(1-\zeta)^{2}+2 \xi^{2}\right]}{45} \cdot \frac{2 \pi P_{0} k^{2} N^{2} n \tilde{\gamma}^{2}}{\hbar} \\
& \times \tanh \left(\frac{\epsilon}{2 k_{\mathrm{B}} T}\right),
\end{aligned}
$$

where again, the two transversally polarized waves, Eqs. (8b) and (드), give the same result.

Finally, for the phonons propagating along the $\langle 110\rangle$ direction we obtain the average scattering rates,

$$
\begin{aligned}
\tau_{\frac{k(\hat{\mathbf{x}}+\hat{\mathbf{y}})}{\sqrt{2}}, l}^{-1}= & \frac{2+6 \zeta+7 \zeta^{2}+4 \xi^{2}}{15} \cdot \frac{2 \pi P_{0} k^{2} N^{2} n \tilde{\gamma}^{2}}{\hbar} \\
& \times \tanh \left(\frac{\epsilon}{2 k_{\mathrm{B}} T}\right),
\end{aligned}
$$

for the longitudinal wave,

$$
\tau_{\frac{k(\hat{\mathbf{x}} \hat{\mathbf{y}})}{\sqrt{2}}, t, z}^{-1}=\frac{4 \xi^{2}}{15} \cdot \frac{2 \pi P_{0} k^{2} N^{2} n \tilde{\gamma}^{2}}{\hbar} \tanh \left(\frac{\epsilon}{2 k_{\mathrm{B}} T}\right),
$$

for the transversal wave polarized in the $\hat{p}_{1}^{\prime}$ direction, and

$$
\tau_{\frac{k(\hat{\mathbf{x}}+\hat{\mathrm{y}})}{\sqrt{2}}, t, p_{2}^{\prime}}^{-1}=\frac{(\zeta-1)^{2}}{15} \cdot \frac{2 \pi P_{0} k^{2} N^{2} n \tilde{\gamma}^{2}}{\hbar} \tanh \left(\frac{\epsilon}{2 k_{\mathrm{B}} T}\right),
$$

for the transversal wave polarized in the $\hat{p}_{2}^{\prime}$ direction. 
In the STM formalism, with $\delta=2 \gamma_{\sigma} S_{\sigma}-\sigma=l, t-$, the transition rates are [4]

$$
\left(\tau_{\mathbf{k}, \sigma}^{(S T M)}\right)^{-1}=\frac{2 \pi P_{0} k^{2} N^{2} n \gamma_{\sigma}^{2}}{\hbar} \tanh \left(\frac{\epsilon}{2 k_{\mathrm{B}} T}\right),
$$

therefore the Eqs. (111)-(13) give the expressions for the $\gamma_{\sigma}^{2} \mathrm{~s}$ for the phonons propagating in different crystalographic directions. Notice that if we impose the condition for isotropy, $\zeta+2 \xi=1$, all equations (11)-(13) reduce to the isotropic expressions of Ref. [4].

Karen Topp, Robert Pohl, and coworkers (see Ref. [6] and references therein) measured $\gamma$ in the crystalographic directions $\langle 100\rangle$ and $\langle 111\rangle$ of the cubic lattice of Ca stabilized Zirconium. They obtained a ratio between $\gamma_{t}$ in the $\langle 111\rangle$ direction and $\gamma_{t}$ in the $\langle 100\rangle$ direction, of about 1.7. Using this result and Eqs. (11b) and (12b), we obtain a relation between $\zeta$ and $\xi$ :

$$
\left(\frac{1-\zeta}{\xi}\right)^{2}=18.4
$$

Notice that the lower symmetry of the cubic lattice modifies the relation $(1-\zeta)^{2} / \xi^{2}=4$, satisfied in an isotropic medium, into the relation (15). More experimental data would enable one to check this relation for other polarizations or propagation directions and eventually even to calculate the elements of $[R]$. If relation (15) does not hold true for any propagation direction and polarization of the elastic wave, then the distribution of TLS orientations is not isotropic.

\section{CONCLUSIONS}

We applied the formalism introduced in Ref. [4] to describe the interaction of phonon modes (or elastic waves) with the ensemble of two-level systems (TLS) in a disordered cubic crystal. We showed that the interaction is anisotropic and-in the language of the standard tunneling model-the coupling constants $\gamma_{l}$ and $\gamma_{t}$ depend on the phonon propagation direction. We focused our calculations on phonons propagating along the crystalographic directions $\langle 100\rangle,\langle 110\rangle$ and $\langle 111\rangle$, for which we gave explicit expressions for the coupling constants. Using the experimental results of Topp, Pohl, and coworkers (see Ref. [6] and references therein) we compared the $\gamma_{t}$ s corresponding to the $\langle 100\rangle$ and $\langle 111\rangle$ crystalographic directions and from here we obtained a relation between the the two parameters of the model, $\zeta$ and $\xi$, that describe the anisotropy of the interaction. Nevertheless, more experimental results are needed (at least $\gamma_{t}$ in one more direction or a $\gamma_{l}$ ) to fully determine these 
parameters and make prediction about the interaction of the TLS system with phonons propagating in any direction. Having these predictions, one then could draw conclusions about the isotropy of the TLS orientations in the material.

\section{Acknowledgements}

We are grateful to Prof. R. Pohl, Prof. K. A. Topp, and Prof. S. Sahling for very useful and motivating discussions. This work was partially supported by the NATO grant, EAP.RIG 982080.

[1] W. A. Philips, J. Low Temp. Phys. 7, 351 (1972).

[2] P. W. Anderson, B. I. Halperin, and C. M. Varma, Phil. Mag. 25, 1 (1972).

[3] C. Laermans and V. Keppens, Phys. Rev. B 51, 8158 (1995).

[4] D. V. Anghel, T. Kühn, Y. M. Galperin, and M. Manninen, Phys. Rev. B 75, 064202 (2007).

[5] T. Kühn, D. V. Anghel, Y. M. Galperin, and M. Manninen, Phys. Rev. B 76, 165425 (2007), cond-mat/07051936.

[6] K. A. Topp, Ph.D. thesis (1997).

[7] D. V. Anghel, T. Kühn, Y. M. Galperin, and M. Manninen (2007).

[8] B. A. Auld, Acoustic Fields and Waves in Solids, 2nd Ed. (Robert E. Krieger Publishing Company, 1990), ISBN 0-89874-783. 